\title{
Intraoperative Nerve Monitoring in Otolaryngology: A Survey of Clinical Practice Patterns
}

\author{
Stephanie Flukes, Shane S. Ling, Travis Leahy, Chady Sader \\ Department of Ear, Nose and Throat Surgery, Fremantle Hospital, Perth, Australia \\ Email: Stephanie.Flukes@health.wa.gov.au
}

Received October 9, 2012; revised November 16, 2012; accepted November 29, 2012

\begin{abstract}
Introduction: Intraoperative nerve monitoring is used in otolaryngology to assist in identification of nerves at risk. It is hoped that this will lead to lower rates of nerve injury. The objective of this study was to quantify the use of monitoring technology in current clinical practice. Method: An electronic survey was distributed to 376 registered fellows of the Australian Society of Head and Neck Surgery. Results: One-hundred and twenty-five responses were obtained. The majority of respondents report using monitoring at least some of the time during thyroid, parotid, and mastoid surgery $(80 \%, 87 \%$, and $73 \%$ respectively). Predictors of use include experience with intraoperative monitoring during training, and high caseloads in parotid surgery. Practice setting did not predict use. Conclusion: Despite equivocal evidence that intraoperative nerve monitoring is associated with a reduction in nerve injuries, this study demonstrates that the technology is widely used amongst otolaryngologists.
\end{abstract}

Keywords: Monitoring; Intraoperative; Mastoid/Surgery; Parotid Gland/Surgery; Thyroid Gland/Surgery; Physician's Practice Patterns

\section{Introduction}

Nerve injury is a serious complication of thyroid, parotid, and mastoid surgery. Damage to the recurrent laryngeal nerve (RLN) during thyroid surgery can result in voice change, aspiration, and airway compromise. Current literature states the rate of permanent RLN paralysis as $0.5 \%$ - 5\%, and higher in revision cases [1-7]. The facial nerve is at risk in both parotid and mastoid surgery. Injury can result in impaired eye closure, which can lead to corneal ulceration and consequent blindness, as well as cosmetic compromise. The published rate of permanent nerve palsy is $0.1 \%-1 \%$ in mastoid surgery [8-10] and $1 \%$ $2 \%$ in parotid surgery $[11,12]$.

Avoidance of intraoperative nerve injury is of paramount importance in order to reduce patient morbidity. In addition, both RLN and facial nerve paralysis are common reasons for litigation following otolaryngology surgery $[13,14]$.

Various techniques have been described to identify nerves at risk. Whilst it is generally accepted that direct visualisation of the nerve is the gold standard, intraoperative nerve monitoring is being used increasingly as an adjunct to help identify the nerve. The use of intraoperative nerve monitoring has been previously reported in the UK and USA [15-18]. To date, no such literature exists to describe practice in other parts of the world. As with many new technologies, the prevalence of nerve monitoring has changed over time. The aim of this study was to determine current usage patterns for intraoperative nerve monitoring and, therefore, inform the surgical community regarding current clinical practice.

\section{Methods}

Ethics approval was obtained from the Western Australian South Metropolitan Area Health Service Human Research Ethics Committee. An electronic questionnaire was designed using SurveyMonkey ${ }^{\mathrm{TM}}$ software

(http:// www.surveymonkey.com/). The survey focused on prevalence and predictors of intraoperative nerve monitoring in thyroid, parotid, and mastoid surgery. Questions encompassed surgeon demographics and training background, current practice setting and caseload, attitudes towards intraoperative nerve monitoring and use of this technology (Appendix 1). An email containing a link to the online survey was sent to all fellows of the Australian Society of Head and Neck Surgery (ASOHNS) using ASOHNS membership data. The survey was available for completion over a period of five weeks, with a reminder email sent after three weeks had elapsed. Deidentified results were collected in a centralised database available via the SurveyMonkey ${ }^{\mathrm{TM}}$ platform. Proportional statistics were calculated using the Wald method to obtain normal approximation intervals. Strength of 
association between the use of monitoring devices and the dependant variables of training, practice setting, and caseload were quantified with odds ratios. Data was processed using Microsoft Excel ${ }^{\mathrm{TM}}$ software.

\section{Results}

Surveys were distributed to all 376 registered fellows of ASOHNS and 125 completed responses were received, representing a $33 \%$ response rate.

\subsection{Demographics}

Duration of ASOHNS fellowship ranged from less than 12 months to 50 years, with a mean of 17 years. With regards to practice setting, 96 respondents worked in both public and private, 20 worked in private only, and 9 worked in public only.

\subsection{Usage Patterns}

Forty-five respondents stated that they regularly performed thyroid surgery, 84 performed parotid surgery, and 112 performed mastoid surgery. Surgeons who reported performing each type of surgery were questioned on their annual caseload (Figure 1) and use of intraoperative nerve monitoring (Figure 2). Selective or routine use of monitoring technology was reported by $80 \%$ of thyroid surgeons $(36 / 45=0.80,95 \%$ confidence interval [CI] $0.68=0.92), 87 \%$ of parotid surgeons $(73 / 84=0.87$, $95 \%$ CI $0.80-0.94)$, and $73 \%$ of mastoid surgeons $(82 / 112=0.73,95 \%$ CI $0.65-0.81)$.

\subsection{Predictors of Use}

Surgeons were questioned regarding their training background and current clinical practice to determine whether these factors influenced the use of intraoperative nerve monitoring (Table 1). Experience with intraoperative nerve monitoring during training was common; $12 \%$ of respondents had used it routinely $(15 / 125=0.12 ; 95 \% \mathrm{CI}$ $0.06-0.18), 60 \%$ had used it sometimes $(75 / 125=0.60$; $95 \%$ CI $0.51-0.69)$; and only $28 \%(35 / 125=0.28 ; 95 \%$ CI 0.20 - 0.36) had never used monitoring during training. Respondents were significantly more likely to use the technology in their current clinical practice if they had used it in training. This was true for those performing thyroid surgery (odds ratio [OR] 13.60; $P<0.001$ ), parotid surgery (OR 4.24; $P=0.03$ ), and mastoid surgery (OR 4.37; $P<0.001$ ). Usage was also compared between high caseload surgeons (those performing greater than 50 cases per year) and low caseload surgeons (those performing fewer than 50 cases per year). High caseload was found to be a predictive factor for mastoid surgeons (OR 36.00; $P<0.001$ ) but not for thyroid or parotid surgeons. Public versus private practice setting did not pre- dict use.

\subsection{Rationale for Monitoring}

Surgeons were questioned on their reasons for using

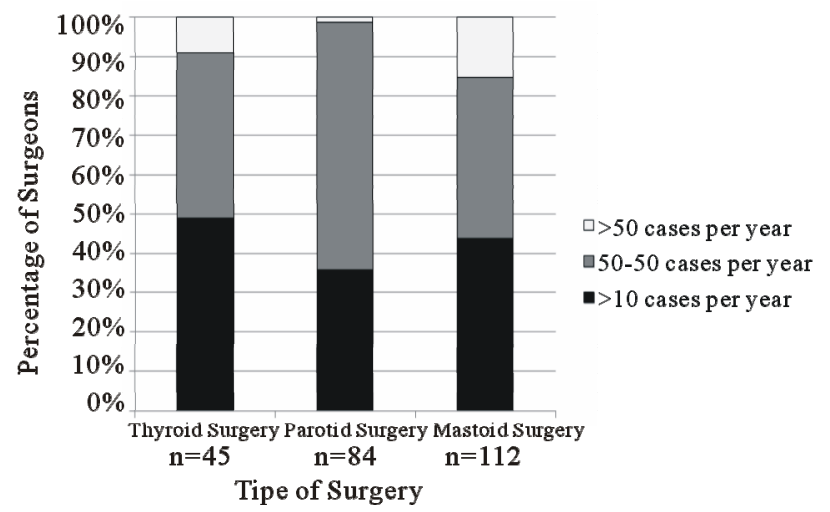

Figure 1. Annual caseload of thyroid, parotid and mastoid surgeries amongst survey respondents. $n$ represents the number of respondents who reported performing each type of surgery.

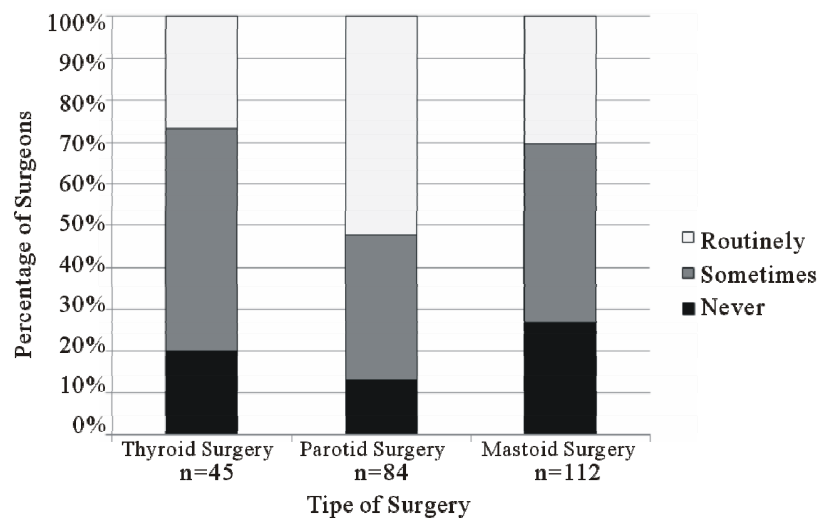

Figure 2. Frequency of use of intraoperative nerve monitoring technology for thyroid, parotid and mastoid surgeries as reported by survey respondents. $n$ represents the number of respondents who reported performing each type of surgery.

Table 1. Influence of surgeon training background, practice setting, and caseload on the use of intraoperative nerve monitoring for thyroid, parotid and mastoid surgeries. The predictive value of each factor is represented as an odds ratio with confidence intervals provided in parentheses. Statistically significant results are highlighted with an asterisk; * represents $\mathbf{p}<0.05$, ** represents $\mathbf{p}<0.001$.

\begin{tabular}{cccc}
\hline \multirow{2}{*}{$\begin{array}{c}\text { Surgeon } \\
\text { background }\end{array}$} & \multicolumn{3}{c}{ Type of surgery } \\
\cline { 2 - 4 } & Thyroid surgery & Parotid surgery & Mastoid surgery \\
\hline Use of monitoring & 13.60 & 4.24 & 4.37 \\
during training & $(1.95-94.61)^{* *}$ & $(1.11-16.15)^{*}$ & $(1.79-10.69)^{* *}$ \\
High caseload & 1.33 & 2.33 & 36.00 \\
Public hospital & $(0.12-14.58)$ & $(0.09-61.11)$ & $(4.61-287.70)^{* *}$ \\
practice setting & $(0.15-160$ & 0.20 & 0.36 \\
\hline
\end{tabular}


intraoperative nerve monitoring (Figure 3). The most common reasons given were to increase safety, to help identify the nerve, and medico-legal protection.

\section{Discussion}

The primary purpose of intraoperative nerve monitoring is to reduce the risk of inadvertent nerve injury. Nerve monitoring may provide early warning of excessive retraction or pressure on the nerve, as well as aid in its localisation. In reality, evidence for a reduction in nerve injury rates with intraoperative monitoring is equivocal. In the case of thyroid surgery, numerous studies have examined the rate of transient and permanent RLN palsy with and without monitoring. Whilst some have shown small benefits in specific subgroups [5,6], none have found an overall statistically significant difference between nerve injury rates in monitored and unmonitored cases [4-6,19-23]. The largest review of the literature is that performed by Higgins et al. in 2011. This meta-analysis of 47 clinical trials evaluated a total 64,699 nerves at risk and found that monitoring made no difference to the rate of transient or permanent RLN paresis [7].

The evidence for the benefit of intraoperative nerve monitoring in parotid surgery is stronger. Makeieff et al. found, in the setting of parotidectomy for recurrent pleomorphic adenoma, monitoring led to significantly lower rates of facial nerve paresis in the monitored group [24]. In addition, operating time was shorter in the monitored group. Terrell et al. found a significant reduction in temporary paresis rates amongst monitored patients undergoing parotidectomy, but no difference in permanent paresis [25]. Reilley et al., however, found no such association [26].

Literature examining the use of monitoring in mastoid surgery is scarce. Anecdotal evidence suggests it may assist in nerve identification [27,28]. Whether this translates into a decreased rate of nerve injury remains unclear.

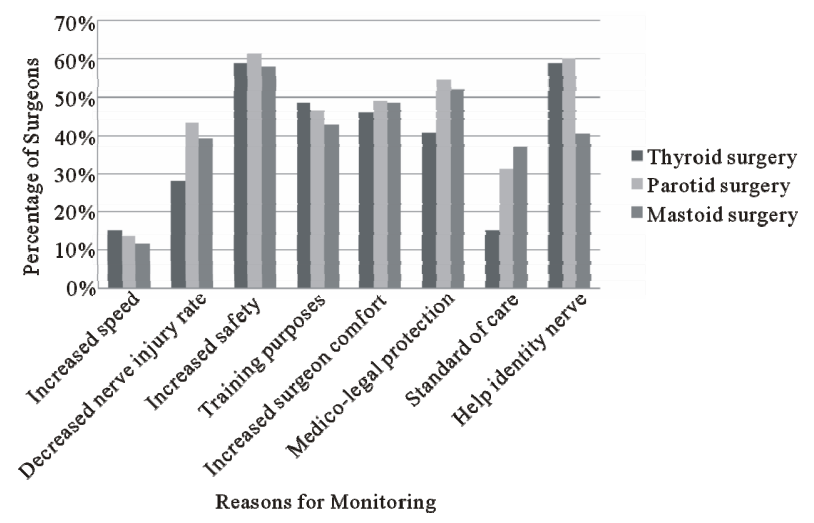

Figure 3. Reasons given by survey respondents for use of intraoperative nerve monitoring in thyroid, parotid, and mastoid surgery.
Another potential benefit of intraoperative nerve monitoring is a reduction in operating time, particularly in revision cases in which nerves can be difficult to identify. This is offset by the increased time taken to set up the device. Other disadvantages include the cost associated with the monitor and a small risk of harm to the patient. Haenggeli reported three cases of facial skin burns during facial nerve monitoring in parotidectomies [29]. There is a theoretical risk of neuropraxia as occurs with direct nerve stimulation; however, this has not been observed in continuous nerve monitoring [30]. Perhaps most importantly, there is a fear that overreliance on intraoperative nerve monitoring can lead to complacency.

Despite a lack of evidence for its use, intraoperative nerve monitoring has been widely adopted by otolaryngologists. Hopkins et al. surveyed 409 ENT surgeons in the UK and found that nerve monitoring was used by $24 \%$ of those performing thyroid surgery, $90 \%$ in parotid surgery, and $51 \%$ in mastoid surgery [15]. Lowry et al. examined use of facial nerve monitoring in parotid surgery in the USA by surveying 1548 otolaryngologists and found that $60 \%$ used monitoring at least some of the time [16]. Both Horne et al. and Sturgeon et al. focused on use of RLN monitoring in thyroid surgery in the USA. Horne et al. surveyed 685 otolaryngologists and found $45 \%$ used monitoring at least some of the time [17]. In contrast, Sturgeon et al. surveyed 117 endocrine surgeons (mostly general surgeons performing thyroid surgery) and found only $37 \%$ used intraoperative nerve monitoring [18].

The findings of this survey reveal a large uptake of intraoperative nerve monitoring technology amongst Australian otolaryngologists who answered this survey. Eighty percent of respondents performing thyroid surgery use monitoring at least some of the time $(+/-12 \%)$, in parotid surgery this figure is $87 \%(+/-7 \%)$, and in mastoid surgery it is $73 \%(+/-8 \%)$. In the case of thyroid and mastoid surgery, this is a higher usage rate than that reflected by the UK and USA studies $[15,17,18]$. In the case of parotid surgery, it is a similar rate to that described in the UK study and higher than that described in the US study $[15,16]$. It should be noted that the abovementioned studies were published between two and 10 years ago, and our reported rates may reflect the changing attitudes towards intraoperative nerve monitoring and its availability to surgeons.

Use of intraoperative nerve monitoring during training was found to be a predictor for use as a consultant surgeon. This suggests that surgeons are more likely to use monitoring if they have a familiarity with the technology. Although there is no conclusive evidence for or against intraoperative nerve monitoring, it is believed by many surgeons to be a useful adjunct. Therefore, it is desirable that surgical trainees gain experience in the use of 
intraoperative monitoring so that they can employ it when they feel it is indicated.

There has been no medicolegal precedent mandating the use of this technology. However, there is a strong perception that usage may limit the liability of the surgeon in the unfortunate event of nerve damage. This attitude is reflected in the survey responses to the question "why do you use intraoperative nerve monitoring?" to which 51\% of respondents replied "for medicolegal protection". Whether or not this belief is true comes down to the issues of "standard of care" with respect to that particular surgery. "Standard of care" is defined by Angelos as "the attention, caution, or prudence that another comparable physician would provide in caring for a patient in a similar circumstance" [31]. Interestingly, the results show that many more respondents consider monitoring to be the standard of care for parotid and mastoid surgery (32\% and $37 \%$ respectively) than do for thyroid surgery $(15 \%)$. As there has been no studies showing a benefit in reducing the rate of nerve damage it cannot be considered the standard of care. However, the awareness of this technology, and the recognition of its increasing use amongst peers, may lead to a perception of its acceptance as the standard of care.

A notable limitation of this survey was the low response rate. This is a common limitation of voluntary surveys and for this reason a reminder email was sent during the data collection phase. Despite this, our response rate was less than that of the other survey-based studies examined [15-18]. As a consequence of this limitation, the results may be confounded by selection bias. For example, those surgeons who regularly use intraoperative nerve monitoring may have been more likely to respond and share their experience. Furthermore, this study was distributed by email and it is possible that surgeons who use nerve monitoring are more comfortable with technology and hence more likely to complete an online survey. The advantages of this survey format are reduction in data handling errors and faster processing of results.

\section{Conclusion}

This survey was performed to determine current clinical practice patterns with regards to use of intraoperative nerve monitoring. The results reveal that the vast majority of consultant surgeons in Australia have adopted the technology for at least some of their cases. This is true for thyroid, parotid, and mastoid operations. These results are clinically significant because of the theoretical reduction in risk of inadvertent nerve injury associated with use of nerve monitoring devices. Although there is no conclusive evidence supporting this theory, the high usage rates demonstrated by this survey suggest that consultant surgeons find the technology helpful. It is therefore hoped that increased use of intraoperative nerve monitoring will be associated with improved patient outcomes. As a cautionary note, it must be emphasised that these devices do not compensate for poor surgical technique. Visual nerve identification will continue to be the gold standard for preventing intraoperative nerve damage.

\section{REFERENCES}

[1] J. P. Jeannon, A. A. Orabi, G. A. Bruch, H. A. Abdalsalam and R. Simo, "Diagnosis of Recurrent Laryngeal Nerve Palsy after Thyroidectomy: A Systematic Review," International Journal of Clinical Practice, Vol. 63, No. 4, 2009, pp. 624-629. doi:10.1111/j.1742-1241.2008.01875.x

[2] G. R. Jatzko, P. H. Lisborg, M. G. Muller and V. M. Wette, "Recurrent Nerve Palsy after Thyroid Operations-Principal Nerve Identification and a Literature Review," Surgery, Vol. 115, No. 2, 1994, pp. 139-144.

[3] J. H. Lefevre, C. Tresallet, L. Leenhardt, C. Jublanc, J. P. Chigot and F. Menegaux, "Reoperative Surgery for Thyroid Disease," Langenbeck's Archives of Surgery, Vol. 392, No. 6, 2007, pp. 685-691. doi:10.1007/s00423-007-0201-6

[4] H. Dralle, et al., "Risk Factors of Paralysis and Functional Outcome after Recurrent Laryngeal Nerve Monitoring in Thyroid Surgery," Surgery, Vol. 136, No. 6, 2004, pp. 1310-1322. doi:10.1016/j.surg.2004.07.018

[5] W. F. Chan, B. H. Lang and C. Y. Lo, "The Role of Intraoperative Neuromonitoring of Recurrent Laryngeal Nerve during Thyroidectomy: A Comparative Study on 1000 Nerves at Risk," Surgery, Vol. 140, No. 6, 2006, pp. 866872. doi:10.1016/j.surg.2006.07.017

[6] M. Barczynski, A. Konturek and S. Cichon, "Randomized Clinical Trial of Visualization versus Neuromonitoring of Recurrent Laryngeal Nerves during Thyroidectomy," British Journal of Surgery, Vol. 96, No. 3, 2009, pp. 240-246. doi:10.1002/bjs.6417

[7] T. S. Higgins, et al., "Recurrent Laryngeal Nerve Monitoring versus Identification Alone on Post-Thyroidectomy True Vocal Fold Palsy: A Meta-Analysis," Laryngoscope, Vol. 121, No. 5, 2011, pp. 1009-1017. doi:10.1002/lary.21578

[8] E. L. Nilssen and P. J. Wormald, "Facial Nerve Palsy in Mastoid Surgery," The Journal of Laryngology \& Otology, Vol. 111, No. 2, 1997, pp. 113-116. doi:10.1017/S0022215100136618

[9] J. D. Green Jr., C. Shelton and D. E. Brackmann, "Iatrogenic Facial Nerve Injury during Otologic Surgery," Laryngoscope, Vol. 104, No. 8, 1994, pp. 922-926. doi:10.1288/00005537-199408000-00002

[10] J. S. Greenberg, S. Manolidis, M. G. Stewart and J. B. Kahn, "Facial Nerve Monitoring in Chronic Ear Surgery: US Practice Patterns," Otolaryngology-Head and Neck Surgery, Vol. 126, No. 2, 2002, pp. 108-114. doi: $10.1067 / \mathrm{mhn} .2002 .121861$ 
[11] S. A. Nouraei, et al., "Analysis of Complications Following Surgical Treatment of Benign Parotid Disease," ANZ Journal of Surgery, Vol. 78, No. 3, 2008, pp. 134-138. doi:10.1111/j.1445-2197.2007.04388.x

[12] N. Klintworth, J. Zenk, M. Koch and H. Iro, "Postoperative Complications after Extracapsular Dissection of Benign Parotid Lesions with Particular Reference to Facial Nerve Function," Laryngoscope, Vol. 120, No. 3, 2010, pp. 484-490. doi:10.1002/lary.20801

[13] K. A. Kern, "Medicolegal Analysis of Errors in Diagnosis and Treatment of Surgical Endocrine Disease," Surgery, Vol. 114, No. 6, 1993, pp. 1167-1173.

[14] S. S. Abadin, E. L. Kaplan and P. Angelos, "Malpractice Litigation after Thyroid Surgery: The Role of Recurrent Laryngeal Nerve Injuries, 1989-2009," Surgery, Vol. 148, No. 4, 2010, pp. 718-722. doi:10.1016/j.surg.2010.07.019

[15] C. Hopkins, S. Khemani, R. M. Terry and D. GoldingWood, "How We Do It: Nerve Monitoring in ENT Surgery: Current UK Practice," Clinical Otolaryngology, Vol. 30, No. 2, 2005, pp. 195-198. doi:10.1111/j.1365-2273.2004.00933.x

[16] T. R. Lowry, T. J. Gal and J. A. Brennan, "Patterns of Use of Facial Nerve Monitoring during Parotid Gland Surgery," Otolaryngology-Head and Neck Surgery, Vol. 133, No. 3, 2005, pp. 313-318. doi:10.1016/j.otohns.2005.03.010

[17] S. K. Horne, T. J. Gal and J. A. Brennan, "Prevalence and Patterns of Intraoperative Nerve Monitoring for Thyroidectomy," Otolaryngology—Head and Neck Surgery, Vol. 136, No. 6, 2007, pp. 952-956. doi:10.1016/j.otohns.2007.02.011

[18] C. Sturgeon, T. Sturgeon and P. Angelos, "Neuromonitoring in Thyroid Surgery: Attitudes, Usage Patterns, and Predictors of Use among Endocrine Surgeons," World Journal of Surgery, Vol. 33, No. 3, 2009, pp. 417-425. doi:10.1007/s00268-008-9724-4

[19] T. Friedrich, et al., "[Intraoperative Electrophysiological Monitoring of the Recurrent Laryngeal Nerve in Thyroid Gland Surgery-A Prospective Study]," Zentralbl Chir, Vol. 127, No. 5, 2002, pp. 414-420. doi:10.1055/s-2002-31983

[20] M. L. Robertson, D. L. Steward, J. L. Gluckman and J. Welge, "Continuous Laryngeal Nerve Integrity Monitoring during Thyroidectomy: Does It Reduce Risk of Injury?" Otolaryngology—Head and Neck Surgery, Vol. 131, No. 5, 2004, pp. 596-600. doi:10.1016/j.otohns.2004.05.030

[21] R. L. Witt, "Recurrent Laryngeal Nerve Electrophysio- logic Monitoring in Thyroid Surgery: The Standard of Care?" Journal of Voice, Vol. 19, No. 3, 2005, pp. 497-500. doi:10.1016/j.jvoice.2004.05.001

[22] M. Shindo and N. N. Chheda, "Incidence of Vocal Cord Paralysis with and without Recurrent Laryngeal Nerve Monitoring during Thyroidectomy," Archives of Otolaryngology-Head and Neck Surgery, Vol. 133, No. 5, 2007, pp. 481-485. doi:10.1001/archotol.133.5.481

[23] S. Sari, et al., "Evaluation of Recurrent Laryngeal Nerve Monitoring in Thyroid Surgery," International Journal of Surgery, Vol. 8, No. 6, 2010, pp. 474-478. doi:10.1016/j.ijsu.2010.06.009

[24] M. Makeieff, et al., "Continuous Facial Nerve Monitoring during Pleomorphic Adenoma Recurrence Surgery," Laryngoscope, Vol. 115, No. 7, 2005, pp. 1310-1314. doi:10.1097/01.MLG.0000166697.48868.8C

[25] J. E. Terrell, et al., "Clinical Outcome of Continuous Facial Nerve Monitoring during Primary Parotidectomy," Archives of Otolaryngology — Head and Neck Surgery, Vol. 123, No. 10, 1997, pp. 1081-1087. doi:10.1001/archotol.1997.01900100055008

[26] J. Reilly and D. Myssiorek, "Facial Nerve Stimulation and Postparotidectomy Facial Paresis," OtolaryngologyHead and Neck Surgery, Vol. 128, No. 4, 2003, pp. 530533. doi:10.1016/S0194-5998(03)00089-5

[27] H. Silverstein, S. I. Rosenberg, J. Flanzer and M. D. Seidman, "Intraoperative Facial Nerve Monitoring in Acoustic Neuroma Surgery," American Journal of Otolaryngology, Vol. 14, No. 6, 1993, pp. 524-532.

[28] R. S. Noss, A. K. Lalwani and C. D. Yingling, "Facial Nerve Monitoring in Middle Ear and Mastoid Surgery," Laryngoscope, Vol. 111, No. 5, 2001, pp. 831-836. doi:10.1097/00005537-200105000-00014

[29] A. Haenggeli, M. Richter, W. Lehmann and P. Dulguerov, "A Complication of Intraoperative Facial Nerve Monitoring: Facial Skin Burns," American Journal of Otolaryngology, Vol. 20, No. 5, 1999, pp. 679-682.

[30] J. Brennan, E. J. Moore and K. J. Shuler, "Prospective Analysis of the Efficacy of Continuous Intraoperative Nerve Monitoring during Thyroidectomy, Parathyroidectomy, and Parotidectomy," Otolaryngology-Head and Neck Surgery, Vol. 124, No. 5, 2001, pp. 537-543. doi: $10.1067 / \mathrm{mhn} .2001 .115402$

[31] P. Angelos, "Recurrent Laryngeal Nerve Monitoring: State of the Art, Ethical and Legal Issues," Surgical Clinics of North America, Vol. 89, No. 5, 2009, pp. 1157-1169. doi:10.1016/j.suc.2009.06.010 


\section{APPENDIX: Questionnaire}

1) In what year did you gain your fellowship to the college?

2) Are you fellowship trained in Otolaryngology, Head \& Neck surgery?

3) Did you use Intra-operative Nerve Monitoring (IONM) during your training?

Answer options: never, sometimes, and routinely.

4) In what setting do you predominately practice?

Answer options: public, private, public \& private.

5) Do you regularly perform thyroid surgery? If so, how many?

Answer options: none, <10 per year, 10 - 550 per year, and $>550$ per year.

If answer is more than "none", proceed to parts a) and b).

a) When do you use IONM in these cases?

Answer options: never, sometimes, and routinely.

b) Why do you use IONM in these cases? You may give more than one answer.

Answer options: increased speed, decrease nerve injury rate, increased safety, for training purposes, increased surgeon comfort, medico-legal protection, standard of care, help identify nerve, other.

1) Do you regularly perform parotid surgery? If so, how many?
Answer options: none, <10 per year, 10 - 550 per year, and $>550$ per year.

If answer is more than "none", proceed to parts a) and b).

a) When do you use IONM in these cases?

Answer options: never, sometimes, and routinely.

b) Why do you use IONM in these cases? You may give more than one answer.

Answer options: increased speed, decrease nerve injury rate, increased safety, for training purposes, increased surgeon comfort, medico-legal protection, standard of care, help identify nerve, other.

2) Do you regularly perform mastoid surgery? If so, how many?

Answer options: none, $<10$ per year, 10 - 550 per year, and $>550$ per year.

If answer is more than "none", proceed to parts a) and b).

a) When do you use IONM in these cases?

Answer options: never, sometimes, and routinely.

b) Why do you use IONM in these cases? You may give more than one answer.

Answer options: increased speed, decrease nerve injury rate, increased safety, for training purposes, increased surgeon comfort, medico-legal protection, standard of care, help identify nerve, other. 